\title{
A New Outranking-Based Approach for Assigning Alternatives to Ordered Classes
}

\author{
Murat Köksalan, ${ }^{1}$ Vincent Mousseau, ${ }^{2}$ Özgür Özpeynirci, ${ }^{3}$ Selin Bilgin Özpeynirci ${ }^{4}$ \\ ${ }^{1}$ Department of Industrial Engineering, Middle East Technical University, 06531, Ankara, Turkey \\ ${ }_{2}^{2}$ LAMSADE, Université Paris Dauphine, Place du Maréchal De Lattre de Tassigny, 75775 Paris Cedex 16, France \\ ${ }^{3}$ Department of Logistics Management, İzmir University of Economics, Sakarya Cad. No 156, 35330 Izmir, Turkey \\ ${ }^{4}$ Department of Industrial Systems Engineering, İzmir University of Economics, Sakarya Cad. No 156, 35330 Izmir, Turkey
}

Received 8 January 2007; revised 23 June 2008; accepted 28 June 2008

DOI 10.1002/nav.20315

Published online 21 October 2008 in Wiley InterScience (www.interscience.wiley.com).

\begin{abstract}
We consider the problem of assigning alternatives evaluated on several criteria into ordered categories $C_{1}, C_{2}, \ldots, C_{p}$. This problem is known as the multi-criteria sorting problem and arises in many situations such as classifying countries into different risk levels based on economical and socio-political criteria, evaluating credit applications of bank customers. We are interested in sorting methods that are grounded on the construction of outranking relations. Among these, the Electre Tri method requires defining multidimensional profiles that represent the "frontier" separating consecutive categories $C_{h}$ and $C_{h+1}$, and assigns an alternative to categories according to how it compares to each of the profiles. The explicit specification of the profiles of consecutive categories can be difficult for decision makers. We develop a new outranking based sorting method that does not require the explicit definition of profiles. We instead require the decision maker to assign a subset of reference alternatives to the categories. To assign the remaining alternatives, each such alternative is compared to reference alternatives, and assigned to categories accordingly. (C) 2008 Wiley Periodicals, Inc. Naval Research Logistics 56: 74-85, 2009
\end{abstract}

Keywords: multiple criteria sorting; outranking relations

\section{INTRODUCTION}

When modeling a real world decision problem using multiple criteria decision aid, several problematics (or problem formulations) can be considered. [16] distinguishes three basic problematics: choice, sorting and ranking.

Given a set $A$ of alternatives (or actions), the choice (or selection) problematic consists in a choice of a subset $A^{\prime} \subset A$, as small as possible, composed of alternatives being judged as the most satisficing. Optimization problems are particular cases of a choice problematic where $A^{\prime}$ is restricted to a single alternative. Purchasing a car or a house are such problems. The ranking problematic consists in establishing a preference preorder (either partial or complete) in the set of alternatives $A$. Ranking academic programs is a typical example. Choice and ranking are comparative problem statements in that comparisons among alternatives are required to reach the results.

Correspondence to: M. Köksalan (koksalan@ie.metu.edu.tr)
The sorting problematic consists in formulating the decision problem in terms of a classification so as to assign each alternative from $A$ to one of the predefined categories. The assignment of an alternative $a$ to the appropriate category should rely on the intrinsic value of $a$ (and not on the comparison of $a$ to other alternatives from $A$ ). Typical sorting examples arise in situations such as classifying countries into different risk levels based on economical and sociopolitical criteria and evaluating credit applications of bank customers. For example, credit rating agencies like Standard \& Poor's (S\&P) regularly evaluate countries based on nine criteria. (http://www2.standardandpoors.com/servlet/Satellite? pagename $=$ sp $/$ Page $/$ HomePg). S\&P currently classifies 113 sovereign governments into one of a number of categories between "AAA" and "CC". AAA corresponds to S\&P's estimate of the group of governments having the least risk of not fulfilling their financial obligations. Naturally, these estimates have a huge impact on the potentials of these countries to borrow and on their borrowing conditions. S\&P indicates that they do not use a fixed formula or weights to establish the ratings. 
Ranking academic programs can also be treated as a sorting problem. Koksalan et al. [6] and Keeney et al. [5] argue that characteristics of this problem makes it more suitable as a sorting problem and they mention various shortcomings of the existing procedures. Whether it is treated as a ranking or a sorting problem, the resulting evaluations are very important for the academic programs, especially in terms of the recruitment of students.

The literature proposes various multiple criteria sorting methods: Trichotomic Segmentation [10], N-TOMIC [9], ORCLASS [8], ELECTRE TRI [20], PROAFTN [1], UTADIS [3], a general class of filtering methods [14], rough sets [4] or Koksalan and Ulu methods [19],[7].

In this article, we are interested in the multiple criteria sorting problems when the preference model is grounded on an outranking relation. More precisely, our work is related to a well known existing method called ELECTRE TRI (see ref. [20], [12]). This method uses a majority rule and a veto rule to compare alternatives to the so called profiles that are considered as representative vectors defining categories. Roughly speaking, when an alternative "outperforms" a category profile in terms of the majority of criteria, and is not "inferior" to that profile by a "large margin," then that alternative cannot be assigned to a worse category than the category represented by that profile. The DM has to define weights, thresholds, and profiles in order to operationalize the method. In practice, it is not realistic to assume that the DM would be able to give explicitly the values of these parameters; the natural terms in which the DM usually expresses his/her preferences and expertise are by making assignments of alternatives to typical categories. Inferring the model parameters of ELECTRE TRI through an analysis of assignment examples given by the DM has already been studied either inferring all parameters simultaneously (see ref. [11]) or a subset of parameters only ([13] and [2]). Nevertheless, one difficulty that has not been addressed when implementing ELECTRE TRI consists in defining (either directly or by inference) the profiles representing the categories. In practice, it is not even reasonable to assume the existence of such profiles. Requiring a DM to specify such profiles is not realistic. However, these profiles have played a central role for methods that are based on ELECTRE TRI.

We propose a new method that avoids the use of such profiles, the categories being implicitly defined by assignment examples. The paper is organized as follows. Section 2 briefly reviews the ELECTRE TRI method and studies properties for outranking based sorting methods. We introduce, in Section 3, our new outranking based sorting method. Section 4 provides a demonstration of the approach through illustrative examples. Last section presents conclusions and issues for further research.

\section{ELECTRE TRI AND OUTRANKING BASED SORTING}

\subsection{Notation and Definitions}

We consider the following notation:

- $A=\left\{a_{1}, a_{2}, \ldots, a_{i}, \ldots, a_{m}\right\}$ - a finite set of $m$ alternatives to be assigned to categories,

- $g_{1}, g_{2}, \ldots, g_{j}, \ldots, g_{n}-n$ evaluation criteria, $g_{j}: A \rightarrow$ $\mathbb{R}$ for all $j \in G=\{1,2, \ldots, n\}$, we will assume, without any loss of generality, that preferences increase with the value on each criterion.

- Considering these $n$ criteria, we define the dominance relation $\Delta$ as $a \Delta b \Leftrightarrow g_{j}(a) \geq g_{j}(b), \forall_{j} \in G$, one of the inequalities at least being strict.

- We consider an outranking relation $S$ on $A$, whose semantic is "at least as good as". We denote indifference relation $I$ and the preference relation $P ; I$ and $P$ correspond to the symmetric and asymmetric parts of $S\left(I=S \cap S^{-1}\right.$ and $\left.\left.P=S \cap\right\urcorner S^{-1}\right)$. We also denote $R$ the incomparability relation on $A$ defined as $R=\urcorner S \cap\urcorner S^{-1}$, i.e., $\left.a R b \Leftrightarrow\right\urcorner(a S b)$ and $\urcorner(b S a)$.

- $C_{1}, C_{2}, \ldots, C_{p}-p$ predefined ordered classes, where $C_{h+1} \gg C_{h}$ (》 a complete order on the set of classes), $h=1, \ldots, p-1$, moreover, $H=\{1, \ldots, p\}$,

- $A^{*} \subseteq A$ - a set of reference alternatives for which the decision maker (DM) defines a desired assignment. We denote $C_{h}^{*} \subseteq A^{*}$ the set of reference alternatives assigned by the DM to category $C_{h}, h \in H(C(a)=$ $\left.h, \forall a \in C_{h}^{*}\right)$,

- $C_{>h}^{*}=\bigcup_{h^{\prime}>h} C_{h^{\prime}}^{*}$ and $C_{<h}^{*}=\bigcup_{h^{\prime}<h} C_{h^{\prime}}^{*}$ the set of reference alternatives that are assigned by the DM to category higher (lower, respectively) than $C_{h}$.

- $X_{j}=\left\{x_{j} \in \mathbb{R}: g_{j}\left(a_{i}\right)=x_{j}, a_{i} \in A\right\}$ - the set of all different evaluations on $g_{j}, j \in G$,

- $X=\prod_{j \in G} X_{j}$ the cartesian product of all evaluation scales, $x \in X$ is called an evaluation vector,

- $P$ denotes a strict preference relation on $A$ (asymmetric and irreflexive), $I$ denotes an indifference relation on $A$ (symmetric and reflexive), $R$ denotes an incomparability relation on $A$ (symmetric and irreflexive). These relations are defined such that: $P \cap I=P \cap R=$ $I \cap R=\emptyset$ and $P \cup I \cup R$ is complete,

- $S$ denotes an outranking relation (weak preference relation) defined by $S=P \cup I$.

\subsection{Brief Review of Electre Tri}

In the Electre Tri method (see ref. [20], [12]), the assignment of an alternative a results from the comparison of $a$ with multidimensional profiles defining the limits of the categories. Let us denote $b_{1}, b_{2}, \ldots, b_{p-1}$ the profiles delimiting the $p$ categories $(B=\{1,2, \ldots, p-1\}), b_{h}$ being the 


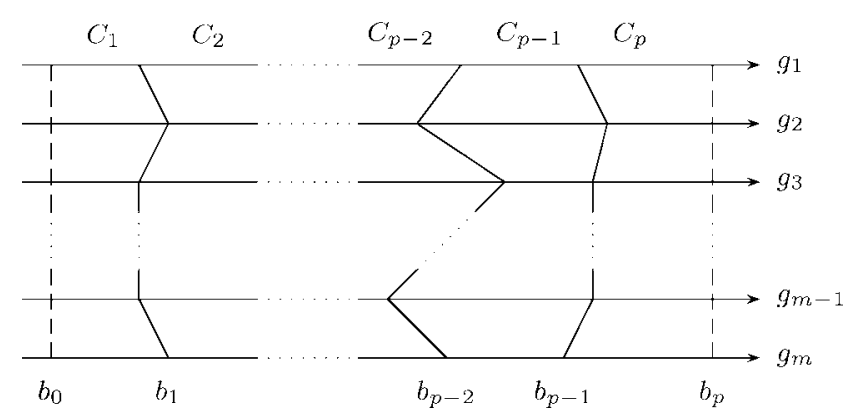

Figure 1. Definition of categories using limit profiles.

upper limit of category $C_{h}$ and the lower limit of category $C_{h+1}, h=1,2, \ldots, p-1$ (see Fig. 1, where the profiles $b_{p}$ and $b_{0}$ correspond to the ideal and anti-ideal alternatives, respectively).

Schematically, Electre Tri assigns alternatives to categories following two consecutive steps:

- construction of an outranking relation $S$ that characterizes how alternatives compare to the limits of categories,

- exploitation of the relation $S$ in order to assign each alternative to a specific category.

Electre Tri builds a valued outranking relation $S$ whose meaning is " $a$ is at least as good as $b_{h}$ ", i.e., defines an index $S\left(a, b_{h}\right) \in[0,1]\left(S\left(b_{h}, a\right)\right.$, resp. $)$ that represents the degree of credibility of the assertion $a S b_{h}$ ( $b_{h} S a$, resp.), $\forall a \in A$, $\forall h \in B$. The assertion $a S b_{h}$ ( $b_{h} S a$, resp.) is considered to be valid if $S\left(a, b_{h}\right) \geq \lambda\left(S\left(b_{h}, a\right) \geq \lambda\right.$, resp. $), \lambda$ being a "cutting level" such that $\lambda \in[0.5,1]$.

Determining $S\left(a, b_{h}\right)$ is done as follows (the value of $S\left(b_{h}, a\right)$ is computed analogously):

1. Compute the partial concordance index $c_{j}\left(a, b_{h}\right)$, $\forall j \in G$ :

$$
c_{j}\left(a, b_{h}\right)=\left\{\begin{array}{l}
0 \quad \text { if } g_{j}\left(b_{h}\right)-g_{j}(a) \geq p_{j}\left(b_{h}\right) \\
1 \text { if } g_{j}\left(b_{h}\right)-g_{j}(a) \leq q_{j}\left(b_{h}\right) \\
\frac{p_{j}\left(b_{h}\right)+g_{j}(a)-g_{j}\left(b_{h}\right)}{p_{j}\left(b_{h}\right)-q_{j}\left(b_{h}\right)} \quad \text { otherwise }
\end{array}\right.
$$

where $q_{j}\left(b_{h}\right)$ and $p_{j}\left(b_{h}\right), j \in G, h \in H$, denote the indifference and preference thresholds that account for the imprecise nature of the evaluations $g_{j}(a)$ (see ref. [18]). $q_{j}\left(b_{h}\right)$ specifies the largest difference $g_{j}(a)-g_{j}\left(b_{h}\right)$ that preserves indifference between $a$ and $b_{h}$ on criterion $g_{j} ; p_{j}\left(b_{h}\right)$ represents the smallest difference $g_{j}(a)-g_{j}\left(b_{h}\right)$ compatible with a preference in favor of $a$ on criterion $g_{j}$.
2. Compute the comprehensive concordance index $c\left(a, b_{h}\right)$ :

$$
c\left(a, b_{h}\right)=\frac{\sum_{j \in F} w_{j} c_{j}\left(a, b_{h}\right)}{\sum_{j \in F} w_{j}}
$$

3. Compute the discordance indices $d_{j}\left(a, b_{h}\right), \forall j \in F$ :

$$
d_{j}\left(a, b_{h}\right)=\left\{\begin{array}{l}
0 \quad \text { if } g_{j}\left(b_{h}\right) \leq g_{j}(a)+p_{j}\left(b_{h}\right) \\
1 \quad \text { if } g_{j}\left(b_{h}\right)>g_{j}(a)+v_{j}\left(b_{h}\right) \\
\in[0,1] \quad \text { otherwise }
\end{array}\right.
$$

where $v_{j}\left(b_{h}\right), j \in G, h \in H$, denote the veto thresholds: an alternative $a$ such that $g_{j}(a) \leq g_{j}\left(b_{h}\right)-$ $v_{j}\left(b_{h}\right)$ cannot outrank $b_{h}$, and an alternative $a^{\prime}$ such that $g_{j}\left(a^{\prime}\right)>g_{j}\left(b_{h}\right)+v_{j}\left(b_{h}\right)$ cannot be outranked by $b_{h}$.

4. Compute the credibility index $S\left(a, b_{h}\right)$ of the outranking relation:

$$
\begin{aligned}
& S\left(a, b_{h}\right)=c\left(a, b_{h}\right) \prod_{j \in \bar{F}} \frac{1-d_{j}\left(a, b_{h}\right)}{1-c\left(a, b_{h}\right)}, \\
& \text { where } \bar{G}=\left\{j \in G: d_{j}\left(a, b_{h}\right)>c\left(a, b_{h}\right)\right\}
\end{aligned}
$$

The values of $S\left(a, b_{h}\right), S\left(b_{h}, a\right)$ and $\lambda$ determine how $a$ and $b_{h}$ compare:

- $S\left(a, b_{h}\right) \geq \lambda$ and $S\left(b_{h}, a\right) \geq \lambda \Rightarrow a S b_{h}$ and $b_{h} S a \Rightarrow$ $a I b_{h}$, i.e., $a$ is indifferent to $b_{h}$,

- $S\left(a, b_{h}\right) \geq \lambda$ and $S\left(b_{h}, a\right)<\lambda \Rightarrow a S b_{h}$ and not $b_{h} S a \Rightarrow a P b_{h}$, i.e., $a$ is preferred to $b_{h}$,

- $S\left(a, b_{h}\right)<\lambda$ and $S\left(b_{h}, a\right) \geq \lambda \Rightarrow$ not $a S b_{h}$ and $b_{h} S a \Rightarrow b_{h} P a$, i.e., $b_{h}$ is preferred to $a$,

- $S\left(a, b_{h}\right)<\lambda$ and $S\left(b_{h}, a\right)<\lambda \Rightarrow \operatorname{not} a S b_{h}$ and not $b_{h} S a \Rightarrow a R b_{h}$, i.e., $a$ is incomparable to $b_{h}$.

Two assignment procedures are then available:

Pessimistic procedure :

a) compare $a$ successively to $b_{i}$, for $i=p, p-1, \ldots, 0$,

b) $b_{h}$ being the first profile such that $a S b_{h}$, assign $a$ to category $C_{h+1}(C(a)=h+1)$.

Optimistic procedure :

a) compare $a$ successively to $b_{i}, i=1,2, \ldots, p$,

b) $b_{h}$ being the first profile such that $b_{h} P a$, assign $a$ to category $C_{h}(C(a)=h)$. 


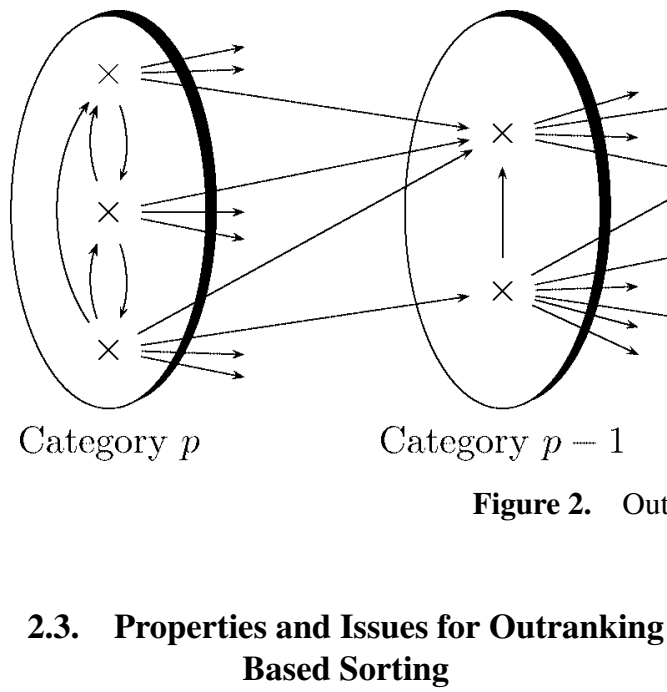

The assignment principle we intend to study is the following:

$$
a S b \Rightarrow C(a)>C(b), \quad \forall a, b \in A
$$

This principle states that "if an alternative $a$ is as good as an alternative $b, a$ should be assigned to a category at least as good as $b$." In other words, this principle forbids that $b$ is assigned to a higher category than $a$ whenever $a S b$. Figure 2, in which no alternative outranks another alternative assigned to a higher category, illustrates this assignment principle. Such assignment principle has been used in [15].

Consider the following proposition:

PROPOSITION 1: Considering the assignment principle (5) holds: If $a_{1} S a_{2}, a_{2} S a_{3}, \ldots, a_{k-1} S a_{k}$ and $a_{k} S a_{1}$, for $a_{1}, a_{2}, \ldots, a_{k} \in A$, then $C\left(a_{1}\right)=C\left(a_{2}\right)=\ldots=C\left(a_{k}\right)$.

PROOF: Consider $k$ alternatives $a_{1}, a_{2}, \ldots, a_{k}$ that are involved in a circuit of the outranking relation $S$, i.e., such that $a_{i} S a_{i_{+} 1}, i=1, . ., k-1$ and $a_{k} S a_{1}$. According to the assignment principle (5) we can deduce that $C\left(a_{1}\right) \geq C\left(a_{2}\right) \geq \ldots \geq$ $C\left(a_{n}\right) \geq C\left(a_{1}\right)$. The only possible assignments respecting these inequalities are : $C\left(a_{1}\right)=C\left(a_{2}\right)=\ldots=C\left(a_{n}\right)$.

This proposition states that the assignment principle (5) implies all alternatives that are in a circuit of the outranking relation $S$ should be assigned to the same category. Hence, the absence of circuits among alternatives assigned to different categories is a necessary condition for the assignment principle (5) to be fulfilled.

It should be noted that the Electre Tri method does not respect such property of absence of circuit among alternatives assigned to different categories. A simple counter-example can be built, considering three alternatives involved in a circuit resulting from a so-called Condorcet effect: 3 alternatives evaluated on 3 criteria (to be maximized) are to be assigned to two categories $C_{1}$ and $C_{2}$. The evaluations of alternatives are given in Table 1.

The criteria weights are equal $\left(w_{1}=w_{2}=w_{3}=\frac{1}{3}\right)$ and $\lambda$ is set to any value between 0.5 and 1 . Moreover, we consider true-criteria $\left(q_{j}\left(b_{1}\right)=p_{j}\left(b_{1}\right)=0, j \in G\right)$, and veto thresholds are set so that no veto phenomenon occur. It is easy to observe that, due to a Condorcet effect, $a_{1} P a_{2}, a_{2} P a_{3}$ and $a_{3} P a_{1}$ holds. Moreover the profile delimiting the categories $C_{1}$ and $C_{2}$ is $b_{1}=(2,0,1)$. Note that $b_{1}$ is the same as $a_{1}$. Hence $a_{1} I b_{1}, b_{1} P a_{2}, a_{3} P b_{1}$ holds. Therefore both Electre Tri assignment rules will assign $a_{1}$ and $a_{3}$ to $C_{2}$, but $a_{2}$ to $C_{1}$.

However, such statements should not be analyzed as shortcomings for the Electre Tri method. Indeed, the principle underlying any sorting method is not to compare alternatives to each other, but rather to analyze each alternative individually so as to define its assignment to a category. In the Electre Tri method, this is performed by comparing each alternative $a$ to reference alternatives that represent the limits of categories.

Hence, it is reasonable to weaken the requirements of the assignment principle (5), so that the implication holds for a subset of alternatives $A^{\prime} \subseteq A$ :

$$
a S b \Rightarrow C(a) \geq C(b), \quad \forall a, b \in A^{\prime} \subseteq A
$$

In Electre Tri, as the ordered categories are defined using profiles $b_{h}$ corresponding to the frontier between category $C_{h}$ and $C_{h+1}$, these $b_{h}$ are such that $b_{h+1} \Delta b_{h}$, i.e., $g_{j}\left(b_{h+1}\right) \geq$ $g_{j}\left(b_{h}\right), \forall j \in G$, one of the inequalities at least being strict, $h=1, \ldots, p-1$. Moreover, [17] imposes an even stronger

Table 1. Condorcet effect.

\begin{tabular}{lccc}
\hline & $g_{1}$ & $g_{2}$ & $g_{3}$ \\
\hline$a_{1}$ & 2 & 0 & 1 \\
$a_{2}$ & 1 & 2 & 0 \\
$a_{3}$ & 0 & 1 & 2 \\
\hline
\end{tabular}

Naval Research Logistics DOI 10.1002/nav 
condition $\left(g_{j}\left(b_{h+1}\right) \geq g_{j}\left(b_{h}\right)+p_{j}\left(b_{h}\right)+p_{j}\left(b_{h+1}\right), \forall j \in\right.$ $G, \forall h \in H)$.

LEMMA 2.1: In the Electre Tri method, when categories are defined such that $g_{j}\left(b_{h+1}\right) \geq g_{j}\left(b_{h}\right)+p_{j}\left(b_{h}\right)+p_{j}\left(b_{h+1}\right)$, $\forall j \in G, \forall h \in H$, no circuits in the outranking relation $S$ can occur involving only a subset of profiles $B^{\prime} \subseteq B=\left\{b_{h}, h=\right.$ $1, \ldots, p-1\}$.

PROOF: Consider the $p-1$ profiles $b_{1}, b_{2}, \ldots, b_{p-1}$ defining the categories in Electre Tri. These profiles ordered by dominance, i.e., defined such that $b_{P-1} \Delta b_{P-2} \Delta \ldots \Delta b_{1}$. As the outranking relation $S$ contains the dominance relation $\Delta, b_{h} S b_{h^{\prime}}$, for all $h, h^{\prime}$ such that $h>h^{\prime}$. Moreover, the definition of categories imposes that $g_{j}\left(b_{h+1}\right) \geq g_{j}\left(b_{h}\right)+$ $p_{j}\left(b_{h}\right)+p_{j}\left(b_{h+1}\right), \forall j \in G, \forall h \in H$, which makes it impossible to have $b_{h^{\prime}} S b_{h}$, for $h, h^{\prime}$ such that $h>h^{\prime}$. Hence, no circuit can occur in $S$ involving only profiles in $B=\left\{b_{h}, h=1, \ldots, p-1\right\}$.

LEMMA 2.2: In the Electre Tri method, when categories are defined such that $g_{j}\left(b_{h+1}\right) \geq g_{j}\left(b_{h}\right)+p_{j}\left(b_{h}\right)+p_{j}\left(b_{h+1}\right)$, $\forall j \in G, \forall h \in H$, no circuits in the outranking relation $S$ can occur involving only one alternative $a \in A$ and a subset of profiles $B^{\prime} \subseteq B \cup\{a\}$ containing more than one profile $\left(\left|B^{\prime}\right| \geq 2\right)$.

PROOF: The profiles separating the categories are defined such that $b_{p-1} \Delta b_{p-2} \Delta \ldots \Delta b_{1}$. Moreover, these profiles are defined such that no alternative $a \in A$ can be indifferent to two consecutive profiles $b_{h}, b_{h+1}, h \in B$, i.e.:

$$
\left.\left.\forall a \in A \text {, it holds: } a I b_{h} \Rightarrow\right\urcorner\left[a I b_{h-1}\right] \text { and }\right\urcorner\left[a I b_{h+1}\right]
$$

Usually imposing (7) is done by posing the following sufficient condition:

$g_{j}\left(b_{h+1}\right) \geq g_{j}\left(b_{h}\right)+p_{j}\left(b_{h}\right)+p_{j}\left(b_{h+1}\right), \quad \forall j \in G, \quad \forall b_{h} \in B$

Considering an alternative $a \in A$ and a profile $b_{h} \in B$, four situations can occur: $a I b_{h}, b_{h} P a, a P b_{h}$ or $a R b_{h}$.

- Suppose $a I b_{h}$, i.e., $a S b_{h}$ and $b_{h} S a$. Consider $b_{h^{\prime}} \in B$, $h^{\prime}>h$. (8) and $a I b_{h}$ implies that $b_{h^{\prime}} P a$. As $b_{h^{\prime}} S b_{h}$ (because $b_{h^{\prime}} b_{h}$ ), alternative $a$ can not be included in a circuit of $S$ involving $b_{h}$ and $b_{h^{\prime}}$. Consider $b_{h}^{\prime \prime} \in B$, $h^{\prime \prime}<h$. (8) and $a I b_{h}$ implies that $a P b_{h^{\prime \prime}}$. As $b_{h} S b_{h^{\prime \prime}}$ (because $b_{h} \Delta b_{h^{\prime \prime}}$ ), alternative $a$ can not be included in a circuit of $S$ involving $b_{h}$ and $b_{h^{\prime \prime}}$.

- Suppose $b_{h} P a$ and consider $b_{h^{\prime}} \in B, h^{\prime}>h$. As $b_{h^{\prime}} \Delta b_{h}$, it holds $b_{h^{\prime}} P a$. Hence, alternative $a$ can not be included in a circuit of $S$ involving $b_{h}$ and $b_{h^{\prime}}$. Consider $b_{h^{\prime \prime}} \in B, h^{\prime \prime}<h$. As $b_{h} \Delta b_{h^{\prime \prime}}$, it holds $b_{h} P b_{h^{\prime}}$.
Hence, alternative $a$ can not be included in a circuit of $S$ involving $b_{h}$ and $b_{h^{\prime \prime}}$.

- Suppose $a P b_{h}$. An argument analogous to the preceding case proves that alternative $a$ can not be included in a circuit of $S$ involving $b_{h}$ and another profile.

- Suppose $a R b_{h}$; alternative $a$ can obviously not be included in a circuit of $S$ involving $b_{h}$ as neither $a S b_{h}$ nor $n_{h} S a$ holds.

Therefore no circuits in the outranking relation $S$ can occur involving only one alternative $a \in A$ and a subset of profiles $B^{\prime} \subseteq B \cup\{a\}$ containing more than one profile.

PROPOSITION 2: When categories are defined such that $g_{j}\left(b_{h+1}\right) \geq g_{j}\left(b_{h}\right)+p_{j}\left(b_{h}\right)+p_{j}\left(b_{h+1}\right), \forall j \in G, \forall h \in H$, Electre Tri method's assignments are compatible with the assignment principle (6) when considering the limit profiles only, i.e., when $A^{\prime}=B$.

PROOF: Because of the dominance among profiles, $S$ constitutes a strict total order on $B: b_{h-1} S b_{h-2} S \ldots S b_{1}$. Electre Tri assigns $b_{h}$ to category $C_{h+1}$. Hence, Electre Tri method's assignments are compatible with the assignment principle (6), when considering the limit profiles only.

PROPOSITION 3: When categories are defined such that $g_{j}\left(b_{h+1}\right) \geq g_{j}\left(b_{h}\right)+p_{j}\left(b_{h}\right)+p_{j}\left(b_{h+1}\right), \forall j \in G, \forall h \in H$, Electre Tri method's assignments are compatible with the assignment principle (6) when considering the limit profiles and any single alternative, i.e., when $A^{\prime}=B \cup\{a\}, a \in A$.

PROOF: According to proposition 2, Electre Tri method's assignments are compatible with the assignment principle (6), when considering the limit profiles only. In order to prove that it remains true when considering $A^{\prime}=B \cup\{a\}, a \in A$, we must consider how $a$ compares to the profiles $b_{h}$ and its comparative assignment, i.e., we need to prove that:

$$
\begin{aligned}
& \text { if } a S b_{h} \text { then } C(a) \geq C\left(b_{h}\right), \quad \forall b_{h} \in B \\
& \text { if } b_{h} S a \text { then } C\left(b_{h}\right) \geq C(a), \quad \forall b_{h} \in B
\end{aligned}
$$

- Electre Tri pessimistic rule assigns $a$ to the highest class for which $a$ outranks the lower profile. Let $b_{h^{\prime}}$ denote the highest profile for which $a S b_{h^{\prime}}$. Then $a$ and $b_{h^{\prime}}$ are both assigned to $C_{h^{\prime}+1}$. As $b_{h^{\prime}}$ is the highest profile for which $a S b_{h^{\prime}}$, $a$ does not outrank any higher profile. Moreover, due to dominance among the ordered profiles, $a$ outranks all profiles lower than $b_{h^{\prime}}$; however each of them are assigned to a category lower than $C_{h^{\prime}+1}$. The optimistic assignment rule assigns $a$ to a category that is higher or equal to the pessimistic assignment. Therefore (9) holds for both assignment rules. 
- Electre Tri optimistic rule assigns $a$ to the lowest class for which the higher profile is preferred to $a$. Let $b_{h^{\prime \prime}}$ denote the lowest profile for which $b_{h^{\prime \prime}} P a$, i.e., $b_{h^{\prime \prime}} S a$ and not $a S b_{h^{\prime \prime}}$. Then $a$ is assigned to $C_{h^{\prime \prime}}$ whereas $b_{h^{\prime \prime}}$ is assigned to $C_{h^{\prime \prime}+1}$. It is possible that $a$ is indifferent to $b_{h^{\prime \prime}-1}$ (but only $b_{h^{\prime \prime}-1}$ as $a$ can not be indifferent to more than one profile), i.e., $b_{h^{\prime \prime}-1} S a$ and $a S b_{h^{\prime \prime}-1}$; in this case $a$ and $b_{h^{\prime \prime}-1}$ are both assigned to $C_{h^{\prime \prime}}$. The pessimistic assignment rule assigns $a$ to a category that is lower or equal to the optimistic assignment. Therefore (10) holds for both assignment rules.

PROPOSITION 4: Even when categories are defined such that $g_{j}\left(b_{h+1}\right) \geq g_{j}\left(b_{h}\right)+p_{j}\left(b_{h}\right)+p_{j}\left(b_{h+1}\right), \forall j \in G$, $\forall h \in H$, Electre Tri method's assignments are not necessarily compatible with the assignment principle (6) when considering all alternatives, i.e., when $A^{\prime}=A$.

PROOF: The example presented in Table 1 provides a counter-example that proves that Electre Tri method's assignments are not necessarily compatible with the assignment principle (6) when considering all alternatives.

\section{A NEW OUTRANKING-BASED SORTING METHOD}

Specifying the category limits through profiles can be a difficult task for the DM. On the other hand, in many decision situations, DMs can easily specify alternatives that are typical examples of a category, say reference alternatives. Therefore, we propose a new outranking-based sorting method that does not require explicit profiles characterizing the multidimensional limits of consecutive categories, but rather represents categories implicitly using reference alternatives.

In this section, we first develop a procedure that captures the circuits, if any, among alternatives assigned by the DM to different categories. We detect the alternatives that cause these circuits and determine which assignments to disregard in order to eliminate the circuits. We then try to assign the remaining alternatives considering the available preference information. We assume that preference and indifference thresholds, therefore partial concordance indices of alternatives are known. We treat criteria weights as unknown.

\subsection{Identifying Assignment Examples Compatible with the Assignment Principle}

To obtain the initial assignments, we ask the DM to assign some alternatives to categories. Let $A^{*}$ be the set of these alternatives. We would like to have approximately equal number of initially assigned alternatives in each category. To achieve this, different approaches can be employed in selecting the alternatives to be presented to the DM for initial assignments. For example, we could use a simplified version of Electre Tri without the veto property. We could use equal weights and suitably chosen profiles and thresholds to come up with some rough assignments. We can then select alternatives from these categories with the expectation that the DM's assignments will roughly be consistent with Electre Tri's assignments. Another approach would be to select the alternatives sequentially utilizing the information obtained from the previous assignments of the DM. We can keep presenting new alternatives to the DM until a desired number of assignments are made to each category. We should emphasize that these are some possible ways of choosing the alternatives in $A^{*}$. The chosen alternatives will be presented to the DM who will make the actual placement of them into categories.

After the DM assigns the given alternatives to categories, we would like to find a large subset of the assignments that do not have circuits. A circuit occurs when two alternatives from different categories outrank each other. There may also be circuits between more than two alternatives. Since there are no profiles for categories, circuits between different categories may exist. We are not concerned about circuits within the same category but we do not allow circuits between categories. In order not to have such circuits, whenever $C\left(a_{q}\right)<C\left(a_{r}\right)$, alternative $a_{q}$ should not outrank alternative $a_{r}$. That is, if an alternative placed in a lower level category outranks an alternative placed in a higher-level category, there is a circuit.

Using model $\left(P_{1}\right)$, we eliminate these circuits (if there exists any) from the reference alternative set. We remove some of the assigned alternatives in order to eliminate these circuits. $\left(P_{1}\right)$ achieves this by maximizing the minimum number of alternatives assigned to any category. This helps in maintaining an approximately equal number of alternatives in each of the categories. After the necessary eliminations from set $A^{*}$, set $A_{1}^{*} \subseteq A^{*}$ is obtained so that there are no circuits between the alternatives in $A_{1}^{*}$.

Decision variables:

$\alpha$ : minimum number of alternatives in a category

$\lambda$ : concordance threshold

$w_{j}$ : weight of criterion $j$

$y_{i}= \begin{cases}1 & \text { if reference alternative } a_{i} \text { is not deleted } \\ 0 & \text { otherwise }\end{cases}$

$\left(P_{1}\right) \operatorname{Max} \alpha+p \sum_{i: a_{i} \in A^{*}} y_{i}$

s.t.

$\alpha \leq \sum_{i: C\left(a_{i}\right)=C_{h}} y_{i}, \quad \forall h \in H$ 
$$
S\left(a_{q}, a_{r}\right)=\sum_{j=1}^{n} w_{j} c_{j}\left(a_{q}, a_{r}\right), \quad \forall a_{q}, a_{r} \in A^{*}
$$

$$
\begin{aligned}
& S\left(a_{q}, a_{r}\right) \leq \lambda-\varepsilon+M\left(1-y_{q}\right)+M\left(1-y_{r}\right), \\
& \quad \forall a_{q}, a_{r} \in A^{*} \ni C\left(a_{q}\right)<C\left(a_{r}\right) \\
& y_{i} \in\{0,1\} \quad \forall i: a_{i} \in A^{*} \\
& w \in \Omega \\
& \lambda \in[0.5,1]
\end{aligned}
$$

where $\rho$ and $\varepsilon$ are arbitrarily small positive constants, and $M$ (big- $M$ ) is an arbitrarily large positive constant. The minimal restrictions on $w$ that define set $\Omega$ are

$$
\begin{aligned}
& \sum_{j=1}^{n} w_{j}=1 \\
& \varepsilon \leq w_{j} \leq 0.5, \quad \forall j
\end{aligned}
$$

If we have additional preference information of the DM, we can incorporate these as additional restrictions in $\Omega$.

Constraints (12) assure that $\alpha$ is less than or equal to the number of assigned alternatives that are not eliminated in each class. These constraints, together with the term, $\alpha$, in the objective function maximize the minimum category size in terms of the retained alternatives assigned by the DM. Constraints (13) define the overall concordance measure in terms of the weighted sum of its components in each criterion. Constraints (14) determine if a circuit exists based on the assignments of $a_{q}$ and $a_{r}$. If a circuit exists, either $a_{q}$ or $a_{r}$ will be eliminated depending on their effects on the objective function.

\subsection{Assigning Alternatives to Categories}

After we eliminate some of the reference alternatives in order to prevent circuits (i.e. after we construct set $A_{1}^{*}$ ) we try to place the unassigned alternatives. In making these assignments, we do not use any profiles. Instead, we utilize the reference alternatives assigned to categories by the DM (i.e. the alternatives in $A_{1}^{*}$ ) as benchmarks to place the remaining alternatives. We denote the alternatives already assigned by the DM as $a_{k}$ and the alternatives we try to place as $a_{i}$.

Decision variables:

$$
y_{i h}= \begin{cases}1 & \text { if alternative } a_{i} \text { is assigned to category } C_{h} \\ 0 & \text { otherwise }\end{cases}
$$

$u_{i h k}=$ Amount of violation in the outranking relation between alternatives $a_{i}$ and $a_{k}$ when $a_{i}$ is assigned to $C_{h}$.
The parameters, $\rho, \varepsilon$, and $M$ are as defined before.

$$
\begin{array}{cl}
\left(P_{2}\right) \text { Min } \quad & \sum_{i} \sum_{h} \sum_{k} u_{i h k}-\rho \lambda \\
\text { s.t. } \quad & \\
& S\left(a_{i}, a_{k}\right) \leq \lambda-\varepsilon+M\left(1-y_{i h}\right), \\
& \forall a_{k} \in C_{>h}, h=1, \ldots, p-1, \forall i \\
& S\left(a_{k}, a_{i}\right) \leq \lambda-\varepsilon+M\left(1-y_{i h}\right), \\
\forall a_{k} \in C_{<h}, h=2, \ldots, p, \forall i \\
& S\left(a_{i}, a_{k}\right) \geq \lambda-M\left(1-y_{i h}\right)-u_{i h k}, \\
\forall a_{k} \in C<h, h=2, \ldots, p, \forall i \\
S\left(a_{k}, a_{i}\right) \geq \lambda-M\left(1-y_{i h}\right)-u_{i h k}, \\
\forall a_{k} \in C_{>h}, h=1, \ldots, p-1, \forall i \\
p \\
\sum_{h=1}^{p} y_{i h}=1, \quad \forall i \\
S\left(a_{q}, a_{r}\right) \leq \lambda-\varepsilon, \\
\forall a_{q}, a_{r} \in A_{1}^{*} \ni C\left(a_{q}\right)<C\left(a_{r}\right) \\
\\
S\left(a_{i}, a_{k}\right)=\sum_{j=1}^{n} w_{j} c_{j}\left(a_{i}, a_{k}\right), \quad \forall i, k \\
\lambda \in[0.5,1] \\
y_{i h} \in\{0,1\}, u_{i h k} \geq 0 \\
w \in \Omega
\end{array}
$$

The model, $\left(P_{2}\right)$, makes sure that any alternative, $a_{i}$, placed by the model does not outrank any alternative, $a_{k}$, placed by the DM to higher-level categories, and is not outranked by any alternative $a_{k}$ placed by the DM to lower-level categories (constraints (21) and (22)). Furthermore, the model tries to have an alternative in a higher-level category to outrank an alternative in a lower-level category between all pairs, and tries to minimize the total magnitude of violations when it is not possible to maintain these relations (constraints (23) and (24)). That is, the $u_{i h k}$ variable measures the magnitude of violation of this outranking relation and the objective function tries to minimize the sum of these violations. The second term in the objective function maximizes the $\lambda$ value as a secondary objective due to its sufficiently small positive coefficient. The constraints (25) make sure that each $a_{i}$ is assigned to exactly one category. (26) force the reference alternatives assigned to lower-level categories not to outrank those assigned to higher-level categories. Constraint (28) specifies the allowable values for $\lambda$. Small values of $\lambda$ are not allowed since it would be too easy for solutions to outrank each other.

To compute the assignment of the nonreference alternatives, we can consider another objective function which minimizes the maximum variable $u_{i h k}$ instead of the sum of these variables. This leads to a program corresponding to an interesting alternative formulation in which the worst deviation is 
considered. This can be achieved by replacing the objective function of $\left(P_{2}\right)$ by:

$$
\operatorname{Min} u-\rho \lambda
$$

and adding constraints

$$
u \geq u_{i h k}, \quad \forall i, h, k
$$

where $u$ represents the maximum violation.

If $\left(P_{2}\right)$ turns out to be infeasible, we may wish to find the placements with minimum number of violations of constraints (21) and (22). Let

$$
z_{i k}= \begin{cases}1 & \begin{array}{c}
\text { if outranking relation between } a_{i} \text { and } a_{k} \\
\text { is violated }
\end{array} \\
0 & \text { otherwise }\end{cases}
$$

$$
\begin{array}{ll}
\text { Min } & \sum_{i} \sum_{k} z_{i k}+\rho \sum_{i} \sum_{h} \sum_{k} u_{i h k} \\
\text { s.t. } & \\
& S\left(a_{i}, a_{k}\right) \leq \lambda-\varepsilon+M\left(1-y_{i h}\right)+M z_{i k}, \\
\forall a_{k} \in C_{>h}, h=1, \ldots, p-1, \forall i \\
& S\left(a_{k}, a_{i}\right) \leq \lambda-\varepsilon+M\left(1-y_{i h}\right)+M z_{i k}, \\
\forall a_{k} \in C_{<h}, h=1, \ldots, p, \forall i \\
\text { Constraints }(23)-(30)
\end{array}
$$

When $z_{i k}$ takes a value of 1 , the above model allows violating the corresponding constraint (34) or (35). The objective function primarily minimizes the number of such violations. Minimizing sum of $u_{i h k}$ variables becomes a secondary objective.

On the other hand, if we want to minimize the total magnitude of violation, or minimize the maximum violation, we can do without the $z_{i k}$ variables. Let $u_{i k}^{\prime}$ be the violation in the outranking relation for the pair $\left(a_{i}, a_{k}\right)$. In this case, we can write the following model.

$$
\begin{array}{ll}
\text { Min } & \sum_{i} \sum_{k} u_{i k}^{\prime}+\rho \sum_{i} \sum_{h} \sum_{k} u_{i h k} \\
\text { s.t. } & \\
& S\left(a_{i}, a_{k}\right) \leq \lambda-\varepsilon+M\left(1-y_{i h}\right)+u_{i k}^{\prime}, \\
\forall a_{k} \in C_{>h}, h=1, \ldots, p-1, \forall i \\
& S\left(a_{k}, a_{i}\right) \leq \lambda-\varepsilon+M\left(1-y_{i h}\right)+u_{i k}^{\prime}, \\
& \forall a_{k} \in C_{<h}, h=2, \ldots, p, \forall i \\
& \text { Constraints }(23)-(30)
\end{array}
$$

(36) minimizes the total violation $u_{i k}^{\prime}$. If we wish to minimize the maximum violation, instead, we then use the following model.

$$
\begin{array}{ll}
\text { Min } & u+\rho \sum_{i} \sum_{h} \sum_{k} u_{i h k} \\
\text { s.t. } & \\
& u \geq u_{i k}^{\prime}, \forall i, k \\
& \text { Constraints }(37),(38),(23)-(30)
\end{array}
$$

\subsection{Finding a Range of Categories}

Another useful information is the range of categories each alternative can be assigned to in light of the set of reference alternatives, $A_{1}^{*}$, obtained from $\left(P_{1}\right)$. To find if alternative $i$ can be assigned to category $h$, we solve a slight variation of $\left(P_{2}\right)$.

Letting $y_{t h^{\prime}}=1$ in $\left(P_{2}\right)$, we can find if it is possible to assign a specific alternative $t$ to a specific category $h^{\prime}$. Let this model be $\left(P 2_{t h^{\prime}}\right)$. If there exists a feasible solution, then it is possible to assign $t$ to $h^{\prime}$ while finding feasible assignments to all remaining alternatives. By solving $\left(P 2_{t h^{\prime}}\right)$ for all $h^{\prime}=1, \ldots, p$, we can find all possible categories of alternative $t$. We can repeat the procedure for all alternatives.

A relaxed version of $\left(P 2_{t h^{\prime}}\right)$ can be obtained as a linear program given as $\left(P 3_{i h}\right)$.

$$
\begin{array}{ccc}
\left(P 3_{i h}\right) \text { Min } \quad 0 & \\
\text { s.t. } & \\
& S\left(a_{i}, a_{k}\right) \leq \lambda-\varepsilon, \quad \forall a_{k} \in C_{>h} \\
& S\left(a_{k}, a_{i}\right) \leq \lambda-\varepsilon, \quad \forall a_{k} \in C_{<h} \\
& S\left(a_{q}, a_{r}\right) \leq \lambda-\varepsilon, \quad \forall a_{q}, \\
& a_{r} \in A_{1}^{*} \ni C\left(a_{q}\right)<C\left(a_{r}\right) \\
& S(a, b)=\sum_{j=1}^{n} w_{j} c_{j}(a, b), \\
& \lambda \in[0.5,1] \\
& w \in \Omega
\end{array}
$$

The constraints of $\left(P 3_{i h}\right)$ are similar to those in previous problems. Since we need to find whether a feasible solution exists or not, the objective function is not relevant and we just set it to a constant value, zero. For each $a_{i}$, we solve $\left(P 3_{i h}\right)$ for all categories. A feasible solution indicates that it might be possible to assign $a_{i}$ to category $h$. When $\left(P 3_{i h}\right)$ does not have a feasible solution, assigning $a_{i}$ to category $h$ would not be consistent with the DM's assignments of reference alternatives.

$\left(P 3_{i h}\right)$ can also be used to reduce the number of integer variables in $\left(P_{2}\right)$ and $\left(P 2_{t h^{\prime}}\right)$. We may define only those $y_{i h}$ variables for which $\left(P 3_{i h}\right)$ indicates a possible assignment. 
Table 2. Reference alternatives.

\begin{tabular}{lcccc}
\hline Program name & Alumni career progress & Diversity & Idea generation & Category of reference alternatives \\
\hline London business school & 68.78 & 62.03 & 59.87 & 5 \\
MIT: Sloan & 60.01 & 24.10 & 85.81 & 4 \\
Yale School of Management & 79.01 & 25.98 & 51.84 & 4 \\
University of North Carolina: Kenan-Flagler & 67.80 & 22.45 & 62.40 & 3 \\
University of Toronto: Rotman & 59.34 & 36.68 & 60.79 & 3 \\
UCLA: Anderson & 55.46 & 21.52 & 74.54 & 2 \\
University of Oxford: Said & 57.04 & 43.49 & 47.12 & 2 \\
University of Maryland: Smith & 55.33 & 25.28 & 64.13 & 1 \\
\hline
\end{tabular}

\section{DEMONSTRATION OF THE APPROACH}

In this section we demonstrate the approach using several problems. We first consider the evaluation problem of MBA programs using actual data. We then use a problem from the banking sector to further demonstrate various aspects of our approach.

\subsection{An Application to Global MBA Rankings}

Here, we demonstrate our method on the MBA program rankings using the Financial Times (FT) 2005 data. Every year, FT announces top 100 global MBA programs. FT uses 20 criteria grouped under three main criteria: alumni career progress, diversity and idea generation.

Ref. [6] studied the MBA program ranking problem for 81 programs of the FT data. We use 30 programs of their list to demonstrate our approach. They create numerical scores that are consistent with the criteria information that are provided in ordinal scale by FT. We use this data set to assign the 30 MBA programs to five categories using three criteria.

We assume that eight programs (reference alternatives) have already been assigned to categories. The scores and categories of the reference alternatives are given in Table 2. Table 3 shows the data for the remaining alternatives that are to be assigned to categories by our models. We use indifference and preference thresholds given in Table 4 . When we solve $\left(P_{1}\right)$, none of the reference alternatives is removed from set $A^{*}$.

We first determine the categories each alternative can be assigned to without causing any circuits. For this purpose, we solve the linear programming model $\left(P 3_{i h}\right)$ for each alternative-category pair. We then solve the mixed integer programming model $\left(P 2_{t h^{\prime}}\right)$. The possible categories of both models for each alternative are given in Table 5. $\left(P 2_{t h^{\prime}}\right)$ reduces the possible number of categories for Cornell University and University of Iowa compared to $\left(P 3_{i h}\right)$. Note that, the size of $\left(P 2_{t h^{\prime}}\right)$ can be reduced by defining binary variables for the assignment of program $i$ only for the possible categories found by $\left(P 3_{i h}\right)$.

Then we solve $\left(P_{2}\right)$ and obtain the assignments given in the last column of Table 5. Optimal weight set is found as
$0.362,0.276$, and 0.362 for the three criteria, and $\lambda$ is found to be 0.638 . The size of $\left(P_{2}\right)$ can also be reduced like $\left(P 2_{t h^{\prime}}\right)$ using the results of $\left(P 3_{i h}\right)$.

Table 3. Alternative scores.

\begin{tabular}{|c|c|c|c|}
\hline Program name & $\begin{array}{c}\text { Alumni } \\
\text { career } \\
\text { progress }\end{array}$ & Diversity & $\begin{array}{c}\text { Idea } \\
\text { generation }\end{array}$ \\
\hline Carnegie Mellon: Tepper & 54.02 & 18.69 & 71.93 \\
\hline $\begin{array}{l}\text { Columbia Business } \\
\text { School }\end{array}$ & 83.44 & 32.94 & 71.63 \\
\hline $\begin{array}{l}\text { Cornell University: } \\
\text { Johnson }\end{array}$ & 60.54 & 30.31 & 55.99 \\
\hline Duke University: Fuqua & 64.05 & 27.25 & 64.68 \\
\hline Esade Business School & 54.61 & 60.15 & 14.45 \\
\hline $\begin{array}{l}\text { Harvard Business } \\
\text { School }\end{array}$ & 77.84 & 29.05 & 93.91 \\
\hline Iese Business School & 61.99 & 54.61 & 49.04 \\
\hline Insead & 56.59 & 70.74 & 65.45 \\
\hline $\begin{array}{l}\text { New York University: } \\
\text { Stern }\end{array}$ & 68.24 & 26.74 & 80.5 \\
\hline $\begin{array}{l}\text { Northwestern } \\
\text { University: Kellogg }\end{array}$ & 68.68 & 24.52 & 72.43 \\
\hline $\begin{array}{l}\text { Rotterdam School of } \\
\text { Management }\end{array}$ & 51.2 & 52.91 & 42.98 \\
\hline Stanford University GSB & 76.77 & 28.52 & 81.8 \\
\hline UC Berkeley: Haas & 61.26 & 31.04 & 73.69 \\
\hline $\begin{array}{l}\text { University of Chicago } \\
\text { GSB }\end{array}$ & 75.64 & 25.37 & 77.73 \\
\hline $\begin{array}{l}\text { University of Iowa: } \\
\text { Tippie }\end{array}$ & 58.25 & 26.25 & 46.81 \\
\hline $\begin{array}{l}\text { University of Michigan: } \\
\text { Ross }\end{array}$ & 63.23 & 28.36 & 69.61 \\
\hline $\begin{array}{l}\text { University of } \\
\text { Pennsylvania: Wharton }\end{array}$ & 77.11 & 32.51 & 91.59 \\
\hline $\begin{array}{l}\text { University of Rochester: } \\
\text { Simon }\end{array}$ & 60.25 & 29.6 & 49.25 \\
\hline $\begin{array}{l}\text { University of Virginia: } \\
\text { Darden }\end{array}$ & 76.49 & 19.05 & 37.68 \\
\hline $\begin{array}{l}\text { University of Western } \\
\text { Ontario: Ivey }\end{array}$ & 50.04 & 40.55 & 53.55 \\
\hline $\begin{array}{l}\text { Vanderbilt University: } \\
\text { Owen }\end{array}$ & 64.67 & 26.33 & 35.14 \\
\hline $\begin{array}{l}\text { York University: } \\
\text { Schulich }\end{array}$ & 57.03 & 50.73 & 47.55 \\
\hline
\end{tabular}


Table 4. Preference and indifference thresholds.

\begin{tabular}{lccc}
\hline & $\begin{array}{c}\text { Alumni career } \\
\text { progress }\end{array}$ & Diversity & $\begin{array}{c}\text { Idea } \\
\text { generation }\end{array}$ \\
\hline Indifference threshold & 0.35 & 0.7 & 0.5 \\
Preference threshold & 3 & 3 & 3 \\
\hline
\end{tabular}

$\left(P_{2}\right)$ places each nonreference alternative $a$ to a category such that $a$ does not outrank a reference alternative of a better category. For example, consider two programs: University of Pennsylvania: Wharton $(W)$ and London Business School $(L)$. According to the optimal criteria weights and the $\lambda$ value found in $\left(P_{2}\right)$, the concordance and outranking relations of these two programs can be calculated as follows:

$$
\begin{aligned}
& g_{1}(L)-g_{1}(W)=68.78-77.11 \\
&=-8.33<q_{1}=0.35 \Rightarrow c_{1}(W, L)=1 \\
& g_{2}(L)-g_{2}(W)=62.03-32.51 \\
&=29.52>p_{2}=3 \Rightarrow c_{2}(W, L)=0 \\
& g_{3}(L)-g_{3}(W)=59.87-91.59 \\
&=-31.72<q_{3}=0.5 \Rightarrow c_{3}(W, L)=1 \\
& S(W, L)=\sum_{i=1}^{3} w_{i} c_{i}(W, L)=0.362+0+0.362=0.724 \\
& g_{1}(W)-g_{1}(L)=77.11-68.78 \\
&=-8.33>p_{1}=3 \Rightarrow c_{1}(L, W)=0 \\
& g_{2}(W)-g_{2}(L)=32.51-62.03 \\
&=29.52<q_{2}=0.7 \Rightarrow c_{2}(L, W)=1 \\
& g_{3}(W)-g_{3}(L)=91.59-59.87 \\
&=31.72>p_{3}=3 \Rightarrow c_{3}(L, W)=0 \\
& S(W, L)=\sum_{i=1}^{3} w_{i} c_{i}(W, L)=0+0.276+0=0.276 \\
& S(W, L)=0.724 \geq \lambda \Rightarrow W \text { outranks } L \\
& S(L, W)=0.276<\lambda \Rightarrow L \text { does not outrank } W
\end{aligned}
$$

Therefore, $W$ cannot be assigned to a category worse than that of $L$. Since $L$ is a reference alternative placed in the best category, $W$ should also be assigned to this category in order not to violate assignment principle (5). It should, however, be noted that the solution provided by $\left(P_{2}\right)$ guarantees principle (5) when considering the comparison of a single nonreference alternative to reference alternatives. As mentioned before, there may be circuits among nonreference alternatives.

Although it is neither necessary nor possible to guarantee preventing circuits among nonreference alternatives with an outranking-based methodology, it is easy to identify the existence of such circuits. A variation of our approach would be to identify those circuits and present them to the DM as feedback. If the DM wishes to prevent some of the circuits, the corresponding alternatives may be added to $A^{*}$ and the whole process can be repeated. In this problem the assignment principle (5) is violated in five of the 231 pairs of nonreference alternatives, leading to circuits involving these five pairs.

In this problem there are 22 schools, each of which is assigned to one of the five categories. Hence $(P 2)$ has 110 binary variables. When we solve $(P 2)$ directly, it takes 1.23 CPU seconds with CPLEX 8.1 on a Pentium $4,2.80 \mathrm{GHz}$ computer with 520 MB RAM. As Table 5 indicates, $\left(P 3_{i h}\right)$ narrows down the possible assignments and we see that 30 of the 110 assignments are not feasible. When we incorporate this information into $(P 2)$, the total CPU time of $\left(P 3_{i h}\right)$ and $(P 2)$ turns out to be $0.66 \mathrm{~s}$.

\subsection{Some Computational Results}

To further demonstrate the performance of our approach, we consider a sorting problem which corresponds to a realworld decision problem in the banking sector [20]. However, due to the confidentiality of the data, the precise context of the problem had not been explained. In this application, the DM considers a new set of alternatives each day. The sorting model aims at distinguishing alternatives so as to eliminate those considered as unsuitable with respect to some predefined norm; at a second stage, a preference ranking of the remaining alternative was build; the final choice was made

Table 5. Results of models $P 3_{i h}, P 2_{i h}$ and $P 2$.

\begin{tabular}{llll}
\hline Program name & \multicolumn{1}{c}{$P 3_{i h}$} & \multicolumn{1}{c}{$P 2_{i h}$} & $P 2$ \\
\hline Columbia Business School & 4,5 & 4,5 & 5 \\
Harvard Business School & 4,5 & 4,5 & 5 \\
New York University: Stern & $3,4,5$ & $3,4,5$ & 5 \\
Northwestern University: Kellogg & $3,4,5$ & $3,4,5$ & 5 \\
Stanford University GSB & $3,4,5$ & $3,4,5$ & 5 \\
University of Chicago GSB & $3,4,5$ & $3,4,5$ & 5 \\
University of Pennsylvania: Wharton & 4,5 & 4,5 & 5 \\
Duke University: Fuqua & $1,2,3,4,5$ & $1,2,3,4,5$ & 4 \\
Insead & $2,3,4,5$ & $2,3,4,5$ & 4 \\
UC Berkeley: Haas & $2,3,4,5$ & $2,3,4,5$ & 4 \\
University of Michigan: Ross & $1,2,3,4,5$ & $1,2,3,4,5$ & 4 \\
Iese Business School & $2,3,4,5$ & $2,3,4,5$ & 3 \\
Cornell University: Johnson & $1,2,3,4,5$ & $1,2,3$ & 2 \\
University of Iowa: Tippie & $1,2,3$ & 1,2 & 2 \\
University of Rochester: Simon & $1,2,3$ & $1,2,3$ & 2 \\
Vanderbilt University: Owen & $1,2,3,4$ & $1,2,3,4$ & 2 \\
York University: Schulich & $2,3,4,5$ & $2,3,4,5$ & 2 \\
Carnegie Mellon: Tepper & 1,2 & 1,2 & 1 \\
Esade Business School & $1,2,3,4,5$ & $1,2,3,4,5$ & 1 \\
Rotterdam School of Management & $1,2,3,4,5$ & $1,2,3,4,5$ & 1 \\
University of Virginia: Darden & $1,2,3,4$ & $1,2,3,4$ & 1 \\
University of Western Ontario: Ivey & $1,2,3,4,5$ & $1,2,3,4,5$ & 1 \\
\hline
\end{tabular}


Table 6. Indifference and preference thresholds and weights of criteria for Electre Tri.

\begin{tabular}{lccccccc}
\hline & \multicolumn{9}{c}{ Criteria } \\
\cline { 2 - 7 } & $g_{1}$ & $g_{2}$ & $g_{3}$ & $g_{4}$ & $g_{5}$ & $g_{6}$ \\
\hline Indifference threshold $q_{j}$ & 0.64 & 1.56 & 1.56 & 1.56 & 1.56 & 1.56 \\
Preference threshold $p_{j}$ & 1.28 & 3.17 & 3.17 & 3.17 & 3.17 & 3.17 & 2.04 \\
Weights $w_{j}$ & 0.24 & 0.12 & 0.12 & 0.12 & 0.12 & 0.12 & 0.16 \\
\hline
\end{tabular}

on the basis of this ranking. We are interested here in the first stage, which consists in the elaboration of a sorting model.

This example problem contains 100 alternatives and seven criteria. We consider placing alternatives into three categories, we simulate DM's responses using Electre Tri with parameters given in Table 6, and we try to choose the reference alternatives to represent categories well.

We first tried our approach by choosing two reference alternatives in each category. In this case, the remaining 94 alternatives were placed by our models. We next added one more reference alternative to each category and run the models again to place 91 alternatives. Finally, we repeated the procedure adding one more reference alternative to each category and placing the 88 nonreference alternatives.

In none of the cases did Model $(P 1)$ encounter any circuits. Hence we did not need to eliminate any of the reference alternatives. The results of Model ( $P 2)$ turned out to be very robust. Regardless of the number of reference alternatives, $(P 2)$ placed the alternatives into the same category, with the exception of one alternative. This alternative was placed to category one when using two and four reference alternatives, but to category two when using three reference alternatives. When we compare the results of the assignments with those of Electre Tri, we observe that a vast majority of alternatives are assigned to the same categories in all cases. There were at most six alternatives assigned to different categories by Electre Tri and our approach. Of course, in theory there could be more disagreement since these are two different approaches. In all solutions, the $\lambda$ value turned out to be very close to its lower bound. This is expected since minimizing $u_{i h k}$ values is in conflict with larger $\lambda$ values. Although the assignments to categories were almost the same with our approach using different number of reference alternatives, there was some variation in the resulting weight values.

We also solved the variation of $(P 2)$ that minimizes the maximum violation discussed in Section 3.2. In this case we minimized the maximum $u_{i h k}$ value, rather than the sum of $u_{i h k}$ values. The placements made by the model were identical to the previous case except for one alternative. The alternative that was an exception in the previous case turned out to be an exception here as well. It was placed to category two when using two and three reference alternatives, but to category one when using four reference alternatives.

Another observation we have is regarding the objective function value of the $(P 2)$ problem. It turned out to be very small (around 0.001), indicating that nonreference alternatives in better categories could outrank reference alternatives in worse categories, and reference alternatives in better categories could outrank nonreference alternatives in worse classes, in general. Looking more closely, only around 10 $u_{i h k}$ variables turned out to be nonzero among 1000 to 2000 possible $u_{i h k}$ values for different cases.

The computational difficulty of $(P 2)$ increases with the number of binary variables. Number of binary variables is equal to the number of alternatives to be assigned by the model times the number of categories. To see the computational performance of our algorithm, we conducted a small experiment. We tried 100 and 300 alternatives. For the 300alternative case we randomly generated 200 alternatives in addition to the original 100 alternatives. We selected five reference alternatives in each category and solved each problem twice; once using only $(P 2)$ and once preprocessing with $\left(P 3_{i h}\right)$ first and then using $(P 2)$ for the simplified problem. We present the CPU times we obtained using CPLEX 8.1 solver on a Pentium 4, $2.80 \mathrm{GHz}$ computer with $520 \mathrm{MB}$ RAM in Table 7. The results indicate that the CPU times increase substantially with the number of alternatives, as expected. The effectiveness of $\left(P 3_{i h}\right)$ also increases substantially with the number of alternatives. The problem is manageable for reasonably large number of alternatives using the CPLEX solver directly. It may be possible to further improve the computations by studying the features of the integer programs solved. This is an issue we consider for future research.

\section{CONCLUSIONS AND FURTHER RESEARCH}

In this article, we developed an outranking-based approach to place alternatives into preference-ordered categories. Our

Table 7. Computational results.

\begin{tabular}{lcc}
\hline & \multicolumn{2}{c}{ CPU seconds } \\
\cline { 2 - 3 } $\begin{array}{l}\text { Number of } \\
\text { alternatives }\end{array}$ & $\begin{array}{c}\text { Using }(P 2) \\
\text { only }\end{array}$ & $\begin{array}{c}\text { Using }\left(P 3_{i h}\right) \\
\text { and }(P 2)\end{array}$ \\
\hline 100 & 3.63 & 3.11 \\
300 & 783.63 & 180.95 \\
\hline
\end{tabular}


approach aims to avoid asking difficult preference information from the DM such as weights or category limits (profiles) or the $\lambda$ threshold. We only require the DM to place a small number of reference alternatives into suitable categories. We then place the remaining alternatives with the aid of an integer program that maintains desired relationships between the reference alternatives and the alternatives that are placed by the model. We demonstrate our approach on a small example problem and observe that it performs well on larger problems as well. Studying various aspects of the integer programs is a future research direction that might prove useful to further improve the computation times.

We believe that there is need for further research to simplify the information requirements of outranking-based approaches. Further developments and experimentation will lead to more realistic outranking-based approaches.

Using the veto concept is not straightforward when parameters are not directly specified by the DM. This is a future research area. Extending our approach to the case of multiple decision makers is another future work we intend to do.

\section{ACKNOWLEDGEMENTS}

This work has benefited from the TÜBITAK-CNRS grant no: MISAG-CNRS-5.

\section{REFERENCES}

[1] N. Belacel, Multicriteria assignment method PROAFTN: Methodology and medical application, Eur J Oper Res 125 (2000), 175-183.

[2] L.C. Dias and V. Mousseau, Inferring electre's veto-related parameters from outranking examples, Eur J Oper Res 170 (2006), 172-191.

[3] M. Doumpos, S. Zanakis, and C. Zopounidis, Multicriteria preference disaggregation for classification problems with an application to global investing risk, Decis Sci 32 (2001), 333-385.

[4] S. Greco, B. Matarazzo, and R. Slowinski, Rough sets methodology for sorting problems in presence of multiple attributes and criteria, Eur J Oper Res 138 (2002), 247-259.

[5] R. Keeney, K. See, and D. van Winterfeldt, Evaluating interdisciplinary academic programs: Applications to us graduate decision programs, Oper Res 54 (2006), 813-828.
[6] M. Koksalan, T. Buyukbasaran, O. Ozpeynirci, and J. Wallenius, An approach to ranking with an application to MBA programs, Technical report 06-01, Department of Industrial Engineering, METU, Ankara, Turkey, 2006.

[7] M. Koksalan and C. Ulu, An interactive approach for placing alternatives in preference classes, Eur J Oper Res 144 (2003), 429-439.

[8] O.I. Larichev and H.M. Moskovich, An approach to ordinal classification problems, Int Trans Oper Res 1 (1994), 375385.

[9] R. Massaglia and A. Ostanello, "N-tomic: A support system for multicriteria segmentation problems," Multiple criteria decision support, P. Korhonen, A. Lewandowski, and J. Wallenius (Editors), Springer Verlag, LNEMS 356, Berlin, 1991, pp. 167-174.

[10] J. Moscarola and B. Roy, Procédure automatique d'examen de dossiers fondée sur une segmentation trichotomique en présence de critéres multiples, RAIRO Recherche Operationnelle 11 (1977), 145-173.

[11] V. Mousseau and R. Slowinski, Inferring an ELECTRE TRI model from assignment examples, J Global Optim 12 (1998), $157-174$.

[12] V. Mousseau, R. Slowinski, and P.Zielniewicz, A user-oriented implementation of the ELECTRE TRI method integrating preference elicitation support, Comput Oper Res 27 (2000), 757-777.

[13] A. Ngo The and V. Mousseau, Using assignment examples to infer category limits for the ELECTRE TRI method, JMCDA 11 (2002), 29-43.

[14] P. Perny, Multicriteria filtering methods based on concordance/non-discordance principles, Annals Oper Res 80 (1998), 137-167.

[15] C. Rocha and L.C. Dias, An idea for ordinal sorting based on ELECTRE without category limits, Research Report n 9/2005, INESC, Coimbra, 2005.

[16] B. Roy, Multicriteria methodology for decision aiding, Kluwer Academic, Dordrecht, 1996.

[17] B. Roy and D. Bouyssou, Aide Multicritere à la Décision : Méthodes et Cas, Economica, Paris, 1993.

[18] B. Roy and P. Vincke, Relational systems of preference with one or more pseudo-criteria: Some new concepts and results, Management Sci 30 (1984), 1323-1335.

[19] C. Ulu and M. Koksalan, An interactive procedure for selecting acceptable alternatives in the presence of multiple criteria, Naval Res Logist 48 (2001), 592-606.

[20] W. Yu, Aide multicritére á la décision dans le cadre de la problématique du tri: méthodes et applications, $\mathrm{PhD}$ thesis, LAMSADE, Université Paris Dauphine, Paris, 1992. 